\author{
MILITARY TECHNICAL COLLEGE \\ CAIRO - EGYPT
}

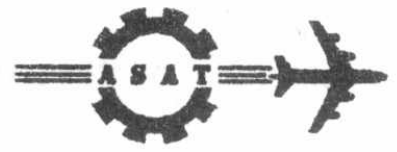

$7^{\text {th }}$ INTERNATIONAL CONF. ON AEROSPACE SCIENCES \& AVIATION TECHNOLOGY

\title{
OPTIMAL DESIGN OF PIEZO-ACTUATORS IN A LAYERED COMPCSITE STRUCTURE
}

\author{
M. M. El-NOMROSSY ${ }^{\star}$, A.A. ISTAFANOS ${ }^{\text {* }}$
}

\begin{abstract}
Optimization of the location and thickness of twin collinear piezoelectric actuators embedded in a layered composite structure is analytically studied. An expression for the effective moment induced by the actuators is derived using a static analysis assuming perfect bonding between layers (i. e, zero bonding thickness).

The optimal actuator position and thickness which maximizes piezo-actuator/ substructure coupling is investigated for an aluminium substructure with lead zirconate titanate (PZT) actuators. Results of this study show that the optimal location for thin actuators is on the surface of the composite. However, for thicker actuator the optimum location is shown to be within the composite structure. An analysis $U_{:}:$actuator thickness shows that the optimal actuator thickness depends on the actuator position. An explanation of this behavior is presented.
\end{abstract}

\section{KEYWORDS}

Optimal Design, Composite, Structure, Piezo-actuators.

\section{NOMENCLATURE}

C Distance from center line of crmposite to center line of actuator.

$\mathrm{d}_{31} \quad$ Piezoelectric strain constant $[\mathrm{m} / \mathrm{V}]$.

E Young's Modulus $\left[\mathrm{N} / \mathrm{m}^{2}\right]$.

h Hali thickness of inner substructure

J Dumm* variable used in the derivation.

$\mathrm{K}$ DL ny variable used in the derivation.

$M$ Bending moment per unit dimension $[\mathrm{N}-\mathrm{m} / \mathrm{m}]$

$t$ Thickness of layer.

* , ** Department of Aeronautics, MTC., Cairo, Egypt. 
T Half thickness of entire composite.

$\mathrm{V}$ Applied voltage.

$x, y, z$ Rectangular coordinate (centered on the composite neutral axis).

\section{Greek}

$\beta \quad$ Non-dimensional property ratio.

$\Delta \quad$ Strain slope $[(\mathrm{m} / \mathrm{m}) / \mathrm{m}]$.

$\varepsilon \quad$ Strain.

$\gamma \quad$ Piezoelectric stress/strain ratio $\left[\mathrm{N} / \mathrm{m}^{2}\right]$.

$\checkmark$ Poisson's ratio.

$\Lambda \quad$ Free piezoelectric strain $[\mathrm{m} / \mathrm{m}]$

$\sigma \quad$ Stress $\left[\mathrm{N} / \mathrm{m}^{2}\right]$.

$\rho \quad$ Non-dimensional actuator thickness and location $[\mathrm{m} / \mathrm{m}]$.

\section{Subscripts}

$\begin{array}{ll}\text { c } & \text { Piezo-actuator location. } \\ \text { E } & \text { Effective. } \\ \text { s1 } & \text { Inner substructure. } \\ \text { s2 } & \text { Outer substructure. } \\ \text { i } & \text { Inner substructure / Piezo-actuator interface. } \\ \text { z } & \text { Piezo-actuator. } \\ 12 & \text { Inner/Outer substructures ratio. } \\ \text { z1 } & \text { Piezo-actuator / Inner substructure ratio. }\end{array}$

\section{INTRODUCTION}

The use of piezoelectric materiales for vibration control of flexible structures has received considrable attention recently. One of the major advantages of piezoelectric materials is that they can be tailored to act as distributed actuators. Furthermore, piezo-actuators have several other attractive characteristics including being inexpensive, lightweight, space efficient, and easily shaped and bonded to surfaces.

Early investigations (Bailey and Hubbard, [1]) into the use of surface-bonded piezoelectric elements as actuators established the viability of piezo-electric materials as actuators for controlling vibrations of flexible structures. This research focused on using surface-bonded piezoelectric actuators as active dampres to reduce the decay time of vibration in beams.

Crawley and de Luis [2] developed expressions for the effective moment generated by both surface-bonded and embedded actuators in one dimensional substructure using a semplified static analysis. Dimitriadis and Fuller [3] extended this analysis to two dimensions in order to study the use of piezo-actuators in actively controlling structuraily radiated / transmitted noise by flexible piates. Kim and Jones [5] also developed a two-dimensional expression for the effective moment using slightly different assumptions of the strain distribution across the composite. They investigated the optimization of actuator thickness for the maximum effective 
moment, and they also demonstrated that thin bonding layers have a negligible effect on the effective moment produced by surface-bonded actuators. Then, Kim and Jones [6] have shown that the effective moment expression using a static analysis is nearly identical to that derived using Love's equation of motion for a composite structure with surface-mounted actuators.

Most of the previous work using piezoelectric materials have concentrated on surface-bonded actuators. However, recently there has been considrable interest in the developing of fully-integrated adaptive systems with actuators and sensors embedded within a composite structure. Embedded transducers have the advantages of being more resistant to surface damage and better protected from the elements than surface-bonded actuators.

In the current work, the optimization of twin embedded collinear actuators is studied. A static analysis is used to derive an effective moment equation for twin embedded collinear piezoelectric actuators in bending. The effective moment equation is nondimensionalized in terms of actuator thickness and actuator position within the layered composite. A discussion of the optimal actuator position and thickness is presented for an aluminium substructure with piezoelectric ceramic actuators.

\section{PIEZO-ACTUATOR/SUBSTRUCTURE COUPLING ANALYSIS}

The motivation for this imagination was to develop a simplified analytical model which can be used to uncover fundamentals of the actuator/substructure interaction. In order to minimize unnecessary complexities which would require solution by numerical methodes, several simplifying assumptions are made. The analytical model employed in this study can be used to illustrate the general trends associated with varying the actuator thickness and location within the layer composite. Thus, the objective of the proposed analytical approach is to provide general guidelines for the optimal design of piezo-actuators embedded within a layered composite structure.

\section{DESCRIPTION OF PLEZOELECTRIC EFFECT}

Piezoelectricity is a phenomenon in which select crystalline materials develop an electric field when subjected to mechanical stress. Piezoelectric materials also exhibit a converse property where by a mechanical deformation in the material is generated by an applied electric field. It is this property which makes piezoelectric materials useful as actuators. The strain developed in the $x-, y-$, and $z$-directions depends on the piezoelectric material constants and the amplitude and sign of the applied electric field. When an embedded piezo-actuator polarized in the thickness ( $\mathrm{z}$ ) direction is excited, surface tractions are generated on the upper and lower surfaces. Twin dctuators symmetrically embedded about the centerline in a substructure, can be excited in-phase (both extend simultaneously), or out-of-phase (one extends, one contracts). Which type of phasing occurs is dependent on the poling direction of the actuators and sign of the applied electric field. In the in-phase configuration, the substructure will experience uniform extension or contraction forces. When the actuators are out-of-phase, the structure will experience bending forces. The out-of- 
phase configuration was analyzed in this investigation because bending vibration is generally more important in noise and vibration control problems.

\section{Derivation of the Effective Moment}

In this section, a derivation of the effective moment generated by twin piezo-electric actuators embedded in a composite laminate is presented.

The following configuration is analyzed : twin small rectangular piezo-actuators symmetrically embedded in a thin layered composite plate and electrically connected to produce bending when a voltage is applied (Figure 1). The structure is symmetric and the inner and outer substructures have in general different mechanical properties. An expression for the effective moment induced by the twin actuators is derived using a static analysis. Previous results (Kim and Jones, [6] ) have shown that for surface-bonded actuators, the effective moment derived using a static analysis is nearly identical to that derived using Love's equations of motion. Thus, small(relative to the overail $\mathrm{x}$ - and $\mathrm{y}$ - dimensions of the composite) piezoelectric patches are assumed in the derivation so that a static can be used and the mass-loading and stiffness effects of the piezo-actuator on the global response of the substructure can be neglected.

A linear strain distribution was assumed across the thickness of the composite. This assumption is valid when the total thickness of the composite is small compared to the radius of curvature of the flexible structure.

A perfect (i.e. zero thickness) bond was assumed between the piezo-actuators and the substrivture. Kim and Jones [5] demonstrated that a thin bonding layer has a negligible effect on the effective moment for surface-bonded actutors $\therefore$

The actuator thickness ( $z$-dimension) is assumed to be small relative to the actuator n-plane ( $\mathrm{x}-$ and $\mathrm{y}^{-}$) dimensions. With this condition, the actuator / substructure coupling is primarily due to the surface tractions on the upper and lower surfaces of the actuator. The edge effects of the actuators on the substructure will generally be small and thus are neglected in this analysis.

For thin elements in bending, the stress in the thickness direction is negligible so Hooke's law for biaxial stress (Dally and Riley, [4]) can be employed :-

$$
\begin{aligned}
& \sigma_{x s}=\frac{E_{s}}{1-v_{s}^{2}}\left(\varepsilon_{x s}+v \varepsilon_{y s}\right) \\
& \sigma_{y s}=\frac{E_{s}}{1-v_{s}^{2}}\left(\varepsilon_{y s}+v \varepsilon_{x s}\right)
\end{aligned}
$$

where the subscripts " $x$ " and " $y$ " refer to the two in-plane directions of the composite plate and the subscript "s" signifies a substructure.

The piezo-actuators and substructure in this study will be assumed to behave isotropically in the $x$ - and $y$-directions. Dimitriadis and Fuller [3] showed that for isotropic piezo-actuators attached to a homogeneous plate, the strains in the $x$ - and $\mathrm{y}$-directions are equal.

Thus, the subscripts $x$ - and $y$ - will be omitted, and the above equations will be simplified to a single equation (3)valid in both the $x$ - and $y$-directions.

The stress-strain relations for the substructure become :- 


$$
\begin{aligned}
& \sigma_{s 1}=\frac{E_{s 1}}{1-v_{s 1}} \varepsilon_{s 1} \\
& \sigma_{s 2}=\frac{E_{s 2}}{1-v_{s 2}} \varepsilon_{s 2}
\end{aligned}
$$

where the $\sigma_{\mathrm{s} 1}$ and $\sigma_{\mathrm{s} 2}$ represent the stress distribution across the inner and outer substructures respectively, as shown in Figure 1.

Because the strain is assumed linear across the thickness direction ( $z)$, the strains in the substructures and the piezo-layer can all be represented as follows :-

$$
\varepsilon(\mathrm{z})=\varepsilon_{\mathrm{s} 1}=\varepsilon_{\mathrm{s} 2}=\varepsilon_{\%}=\Delta \mathrm{z}
$$

where $\Delta$ is the strain slope. The stress distribution across the layered composite thickness will also be linear within each independent layer since the strain is assumed linear across the thickness of the composite. However, the stress distribution will be discontinuous at the interface between substructures and piezo-layer due to differing material properties and, the induced stress generated by the piezo-actuator (Figure 1). In order to simplify the derivation of the effective moment, the stress equation for the substructures and the piezo-layer will be expressed in terms of the interface stress acting on the inner substructure, $\left(\sigma_{i}\right)_{s 1}$. The stress in the inner substructure can be rewritten in terms of the interface stress as :-

$$
\sigma_{\mathrm{s} 1}=\frac{\left(\sigma_{\mathrm{i}}\right)_{\mathrm{s} 1}}{\mathrm{~h}} \mathrm{z}
$$

where $h$ is the half thickness of the inner substructure $\left(h=t_{s l} / 2\right)$.

By combining equations (3), (5), and (6), the strain slope, $\Delta$, can be obtained as a function of the interface stress on the inner substructure as :-

$$
\Delta=\frac{1-v_{s 1}}{E_{s 1}} \frac{\left(\sigma_{t}\right)_{s 1}}{h}
$$

Now, by combining equatios (4),(5) and (7), the stress in the outer substruture can also be represnted in terms of the interface stress as:-

$$
\sigma_{s 2}=\beta_{12} \frac{\left(\sigma_{i}\right)_{s 1}}{h} z
$$

where

$$
\beta_{12}=\frac{E_{s 2}}{E_{s 1}} \frac{1-v_{s i}}{1-v_{s 2}}
$$

For a voltage applied in the z-direction, and assuming an elastically isotropic actuator , the stress-strain relation for the piezo-layer can be written as:- 
where

$$
\sigma_{z}=\frac{l i}{1-v_{z}}(\varepsilon-\Lambda)
$$

Here, $\Lambda$ is the free piezoelectric strain in the actuator which is a function of the applied voltage $(\mathrm{V})$, the actuator thickness $(\mathrm{t}, \mathrm{)}$, and the piezoelectric strain constant $\left(d_{i 1}\right)$. The subscript " $z$ " refers to the piezo-layer

By combining equations (5), (7) and (9), the stress in the piezo-layer can be represented in terms of the interface stress as:

where

$$
\begin{aligned}
& \sigma_{1}=\beta_{i, 1} \frac{\left(\sigma_{i}\right)_{s i}}{h} z-y_{1} \Lambda \\
& \beta_{z 1}=\frac{E_{z}}{E_{i 1}} \frac{1-v_{s 1}}{1-v_{z}} \quad \text { and } \quad y=\frac{E_{z}}{1-v_{z}}
\end{aligned}
$$

The moment equilibrium condition can now be used with equations (6), (8) and (11) to solve for the inner interface stress. Making use of the symmetry about the neutral axis of the composite, the equilibrium condition can be expressed as :-

$$
M=2 \int_{v .1}^{\text {edge }} \sigma z d z=0
$$

or

$$
M=2 \int_{0}^{h} \sigma_{s i} z d z+\int_{h}^{h+1} \sigma_{,} z d z+\int_{i n+t_{2}}^{h+t_{2}+t_{s 2}} \sigma_{s} z d z=0
$$

By substituting the expressions for $\sigma_{11}, \sigma_{1}$, and $\sigma_{52}$ into eqution (12), performing the integrations and solving the equilibrium equation for the inner interface stress yields the following

$$
\left(\sigma_{i}\right)_{s 1}=\frac{3 h\left(2 h t_{,}+t_{i, 2}^{2}\right)}{2\left(h^{3}+\beta_{, 1} K+\beta_{12} J\right)} \gamma \Lambda
$$

where

$$
\begin{aligned}
& K=3 h^{2} t_{1}+3 h t_{;}^{2}+t_{1}^{3} \\
& J=t_{s 2}^{3}+3 t_{\mathrm{s} 2}^{2}(h+t)+3 t_{\mathrm{v} 2}\left(h^{2}+t_{1}^{2}\right)+6 h t_{2} t_{s 2}
\end{aligned}
$$

The effective bending moment applied to the substructures can be represented as the sum of the moments applied to the outer and inner substructures as given below:- 


$$
M_{E}=\int_{-h-t_{z}-t_{12}}^{-h-1,} \sigma_{\mathrm{s}} z d z+\int_{-h}^{h} \sigma_{\mathrm{s} 1} z d z+\int_{h+t_{z}}^{h+t_{1}+t_{s 2}} \sigma_{\mathrm{s} 2} z d z
$$

By substituting the expressions for $\sigma_{\mathrm{s} 1}$ and $\sigma_{\mathrm{s} 2}$ into equation (16) and performing the integrations, a general expression for the effective moment is given by :-

$$
M_{1:}=\frac{\left(2 h t_{\%}+t_{\%}^{2}\right)\left(h^{3}+\beta_{12} J\right)}{\left(h^{3}+\beta_{\%, 1} K+\beta_{12} J\right)} \gamma \Lambda
$$

This expression for the bending moment generated by twin collinear piezo-actuators is a function of the materials properties and the thickness of the substruture layers and the thickness, location, and material properties of the embedded actuators.

For the case when, the inner and outer substructures have the same material properties, hence $\beta_{12}=1$, then the inner interface stress will be :-

$$
\left(\sigma_{i}\right)_{s 1}=\frac{3 h\left(2 h t_{\%}+t_{\%}^{2}\right)}{2\left(h^{3}+\beta_{\%, 1} K+J\right)} \gamma \Lambda
$$

and the effective bending moment

$$
{ }^{4} \mathrm{M}_{\mathrm{E}}=\frac{\left(2 \mathrm{ht}_{7}+\mathrm{t}_{\mathrm{z}}^{2}\right)\left(\mathrm{h}^{3}+\mathrm{J}\right)}{\left(\mathrm{h}^{3}+\beta_{21} \mathrm{~K}+\mathrm{J}\right)} \gamma \Lambda
$$

\section{Non-Dimensionalized Moment Equation}

In order to more understand the behavior of embedded actuators, it was desired to determine the maximum effective moment that can be produced by various thicknesses and locations of actuators within the layered composite.

Also, the moment relationship of equations (17) and (19) are non-dimensionalized to the degree possible to aid in generalizing the results.

The analysis is simplified by assuming a constant thickness composite.

This represents the case where the dimensions of the overall composite are held constant, but the internal arrangement of substructures may be changed.

The actuator thickness may be varied from zero thickness (no actuators ) up to the half thickness of the composite (no substructure). Also, the actuator position within the thickness of the composite may be varied from the case of surface-bonded actuator to the case where the twin actuators lie side by side at the centerline of the composite. It should be noted that some of these limiting cases represent physically impractical cofigurations of the composite structure. Neverthless, the primary objective here is to look at all the theoretically possible cofigurations, since investigating the limiting cases can provide some basic insight into the behavior of the overall structure.

For this analysis, the efective moment equations are written in terms of the actuator position $(C)$ and thickness $\left(t_{7}\right.$ ) by using the following relations: 


$$
\begin{aligned}
& h=C-t_{\%} / 2 \\
& t_{\mathrm{s} 2}=T-h-t_{\%}=T-C-t_{\%} / 2
\end{aligned}
$$

where $\mathrm{C}$ is the distance between centerline of the composite and the centerline of the actuator. The moment equations can now be non-dimensionalized in terms of the composite half thickness $(\mathrm{T})$ by using the following non-dimensional variables

$$
\rho_{1}=\frac{t}{T} ; \quad \rho_{s}=\frac{C}{T}
$$

where the physical limits on $\rho_{\%}$ and $\rho_{c}$ are :-

$$
0 \leq \rho_{\%} \leq 1 \quad \text { and } \quad \frac{\rho_{t}}{2} \leq \rho_{\mathrm{c}} \leq 1-\frac{\rho_{\%}}{2}
$$

After simlification using equations (20) to (23), the effective moment equations (17) and (19) become :-

- For the case of different substructure material properties:-

$\mathrm{m}_{\mathrm{E}}=\frac{\mathrm{M}_{\mathrm{F}}}{\mathrm{T}^{2} \gamma \Lambda}=\frac{2 \rho_{,} \rho_{\mathrm{u}}\left\{8 \beta_{12}+8\left(1-\beta_{12}\right) \rho_{\mathrm{c}}^{3}-12\left(1-\beta_{12}\right) \rho_{\mathrm{c}}^{2} \rho_{\mathrm{z}}+6\left(1-\beta_{12}\right) \rho_{\mathrm{c}} \rho_{\mathrm{z}}^{3}-\left(1-\beta_{12}\right) \rho_{\mathrm{z}}^{3}\right\}}{8 \beta_{12}+8\left(1-\beta_{12}\right) \rho_{\mathrm{c}}^{3}+12\left(2 \beta_{, 1}-1-\beta_{12}\right) \rho_{\mathrm{c}}^{2} \rho_{\mathrm{z}}+6\left(1-\beta_{12}\right) \rho_{\mathrm{c}} \rho_{\mathrm{z}}^{3}+2\left(\beta_{\mathrm{z} 1}-1-\beta_{12}\right) \rho_{\mathrm{z}}^{3}}$

- For the case of similar substructure material properties

$$
m_{1:}=\frac{M_{1:}}{T^{2} \gamma \Lambda}=\frac{2 \rho_{1} \rho_{c}\left(4-12 \rho_{c}^{2} \rho_{z}-\rho_{z}^{3}\right)}{4+12 \rho_{c}^{2} \rho_{z}\left(\beta_{z, 1}-1\right)+\rho_{z}^{3}\left(\beta_{z, 1}-1\right)}
$$

In the following section, this last expression will be used to analyze the optiminal actuator thickness and position.

\section{RESULTS AND DISCUSSION}

The following sections focus on interpreting the character of the effective moment for specific representative actuator/substructure configuration. The first section concerns the optimization of the effective moment with respect to actuator location. This is followed by a discussion of the optimal actuator thickness.

For the subsequent analysis, the layered composite is assumed to be comprised of an aluminum substructure with collinear piezo-ceramic (PZT) actuators embedded in the substructure. The overall thickness of the layered composite is assumed to be $2(\mathrm{~mm})$

The applied electric field strength is assumed to be $100(\mathrm{~V} / \mathrm{mm})$ which approaches the maximum allowable for continuous use of a piezoelectric ceramic material. This gives a free piezoelectric strain $(\Lambda)$ of $2 \times 10^{-5}(\mathrm{~mm} / \mathrm{mm})$ using the piezoelectric strain constant dis of $200 \times 10^{-12}(\mathrm{~m} / \mathrm{V})$. The moduli of aluminium and PZT are assumed to 

be $7.1 \times 10^{10}$ and $6.5 \times 10^{10}\left(\mathrm{~N} / \mathrm{m}^{2}\right)$, with Poisson's ratios of 0.30 and 0.33
respectively.

With the composite thickness and actuator/substructure material properties specified, the effective moment equation becomes a function of two variables:

- the non-dimensional actuator position, $\rho_{\mathrm{c}}$

- the non-dimensional 'actuator thickness, $\rho_{\%}$.

\section{Optimal Actuator Location}

It can be seen from equation (25) that for thin actuators, the maximum moment is achieved when the actuator is positioned on the surface of the composite . However, for thicker actuators, there exists an optimal position within the composite that gives the maximum effective moment. Two specific cases are used to illustrate this point Figure (2) shows the effective moment vs actuator position $\left(\rho_{c}\right)$ for a nondimensional actuator thickness $\rho$, of 0.05 . The figure shows that for thin actuators, the maximum moment occurs at the surface. The moment decreases in an approximately linear fashion as the actuators are moved toward the centerline of the composite.

Quite different relationship exists for thicker actuators. Figure (3) shows the effective moment vs actuator position for an actuator thickness of $\rho_{z}=0.3$. In this case, the maximum effective moment occurs when the actuator is placed within the composite rather than on the surface. It makes sense that as actuator is moved farther away from the neutral axis (toward the surface of the composite), the moment that it can generate increases. Thus it would seem that the effective moment would be a maximum at the surface of the composite.

What this argument fails to account for is that the restoring moment within the actuator layer also increases as the actuators are moved outward. This can be illustrated by revisiting the moment equation (13)

Substituting for $\sigma_{\mathrm{s} 1}, \sigma_{\%}$, and $\sigma_{\mathrm{s} 2}$ gives the following :-

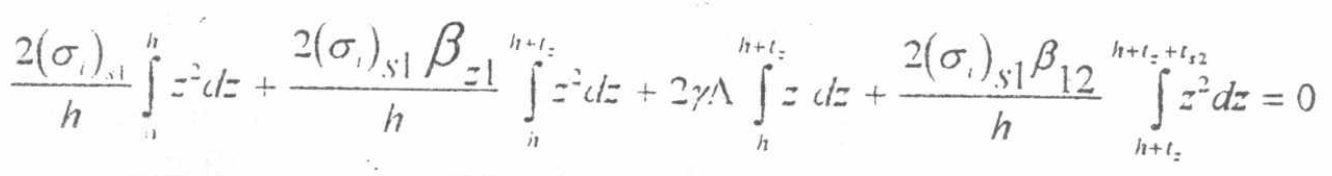

$$
[2]
$$

where the terms $[1],[2],[3]$ and [4] are internal moments within the layered composite representing :-

[1] the restoring moment in the inner substructure.

[2] the restoring moment in the piezo-actuator.

[3] the moment exerted by the piezo-actuator.

[4] the restoring moment in the outer substructure.

Note that the effective moment is simply the moment applied to the substructure layers (terms $[1]+[4])$, and can also be thought of as the moment exerted by the actuator minus the restoring moment in the actuator (terms[3]-[2]).

Figure (4) shows a breakdown of the internal moments in the composite as a function of the non-dimensional actuator position for $\rho_{z}=0.3$. This figure shows that the moment exerted by the actuator [3] follows a linear relationship with the actuator position. The restoring moment within the actuator, [2] follows a higher-order relationship with actuator position. Since the effective moment equals the moment 
generated by the actuator (term[3] above) minus the restoring moment (term[2] above) within the actuator, the effective moment will be a maximum when the restoring moment begins to increase faster than the moment applied by the piezoactuator. This occurs when the slope of the two curves are equal. If the actuators are moved farther toward the surface of the composite, the restoring moment increases faster than the generated moment, resulting in a reduction in the effective moment Thin actuators do not exhibit this behavior when positioned within the composite. This is due to the fact that the restoring moment does not increase fast enough with respect to actuator position, and the slope of the restoring moment never equals the slope of the generated moment within the thickness of the layered composite structure.

\section{Optimal Actuator Thickness}

The optimal actuator thickness behaves in a manner similar to the optimal position As was the case for the optimal position, when the slope of the restoring moment equals the slope of the generated moment of the piezo-actuator, the effective moment will be a maximum. For actuators positioned near the outside edge of the composite (e.g., $\rho_{c}>0.9$ ), the effective moment is maximum when the actuator extends all the way to the outer surface. That is, the actuator cannot be made thick enough for the slope of the restoring, moment to equal the slope of the generated moment, so the maximum value occurs when the thickness is increased to the physical limits of the configuration. The same logic holds for actuators positioned near the ceterline of the composite $\left(\rho_{c}<0.4\right)$. The maximum moment is achieved when the actuator is made thick enough to extend to the centerline of the structure.

For actuators located midway between the centerline and outer edge of the composite, there will exist an optimal thickness where the effective moment is a maximum, and the slope of the restoring and generated moment are equal (Figure5). In this case, the optimal actuator will not extend far enough to reach either the centerline or the outside edge of the structure.

The above analysis gives insight into the optimal actuator configuration for maximum effective moment. However, it should be emphasized that the actuator location and thickness should not be chosen solely on the basis of maximum effective moment. Other important factors should be considered such as the influence of actuator thickness and location on the micromechanics of embedding the actuators in a composite structure. Specifically, the effects on crack formation and propagation in the actuator/substructure interface need to be taken into account before selecting a final design. Other factors should also be considered, such as the case of manufacturing the composite, the availability and cost of actuators, and the effective moment required for the particular application

\section{CONCLUSIONS}

A new expression was derived for the effective moment produced by a pair of piezoactuators embdded in a layered composite. The behavior of an aluminium substructure with embedded PZT actuators was analytically investigated For thick actuators, it was shown that the maximum effective moment is produced for an actuator layer located within the composite structure. It was also shown that for thin actuators the maximum effective moment is achieved when the actuators are 
located at the surface. The optimal actuator thickness was shown to be a function of actuator position, and that actuators located toward the outside edge of the composite should extend all the way to the outer surface for maximum moment Similarly, actuators positioned near the center of the composite should extend to the centerline to maximize the effective moment.

\section{REFERENCES}

[1]Bailey, T. and Hubbard, J.E. "Distributed Piezoelectric-Polymer Active Vibration Control of a Cantilever Beam ", AIAA Journal of Guidance and Control, Vol.8, No.5,pp 605-611,(1985)

[2]Crawley, E.F., and Deluis, J., "Use of Piezoelectric Actuators as Elements of Intelligent Structures", AIAA Journal, Vol.25, No. 10, pp 1373-1385, (1987).

[3]Dimitriadis, E.K., and Fuller, C.R., "Piezoelectric Actuators for Noise and Vibration Control of Thin Plates", $12^{\text {th }}$ Annual ASME Conference on Mechanical Vibration and Noise, Montreal, Canada, (1989).

[4]Dally, J. W., and Riley, W.F., "Experimental Stress Analysis", MacGraw Hill, New York, (1965).

[5]Kim, S.J., and Jones, J.D., "Optimal Design of Piezo-Actuators for Active Noise and Vibration Control", AIAA-90-3925, AIAA $13^{\text {th }}$ Aeroacoustics Conference, Florida, (1990).

[6]Kim, S.J. and Jones, J.D., "Optimization of Piezo-Actuator Substructure Coupling for Active Noise and Vibration Control", Proceedings of the Conference on Recent Advances in Active Control of Sound and Vibration, Virginia, (1991).

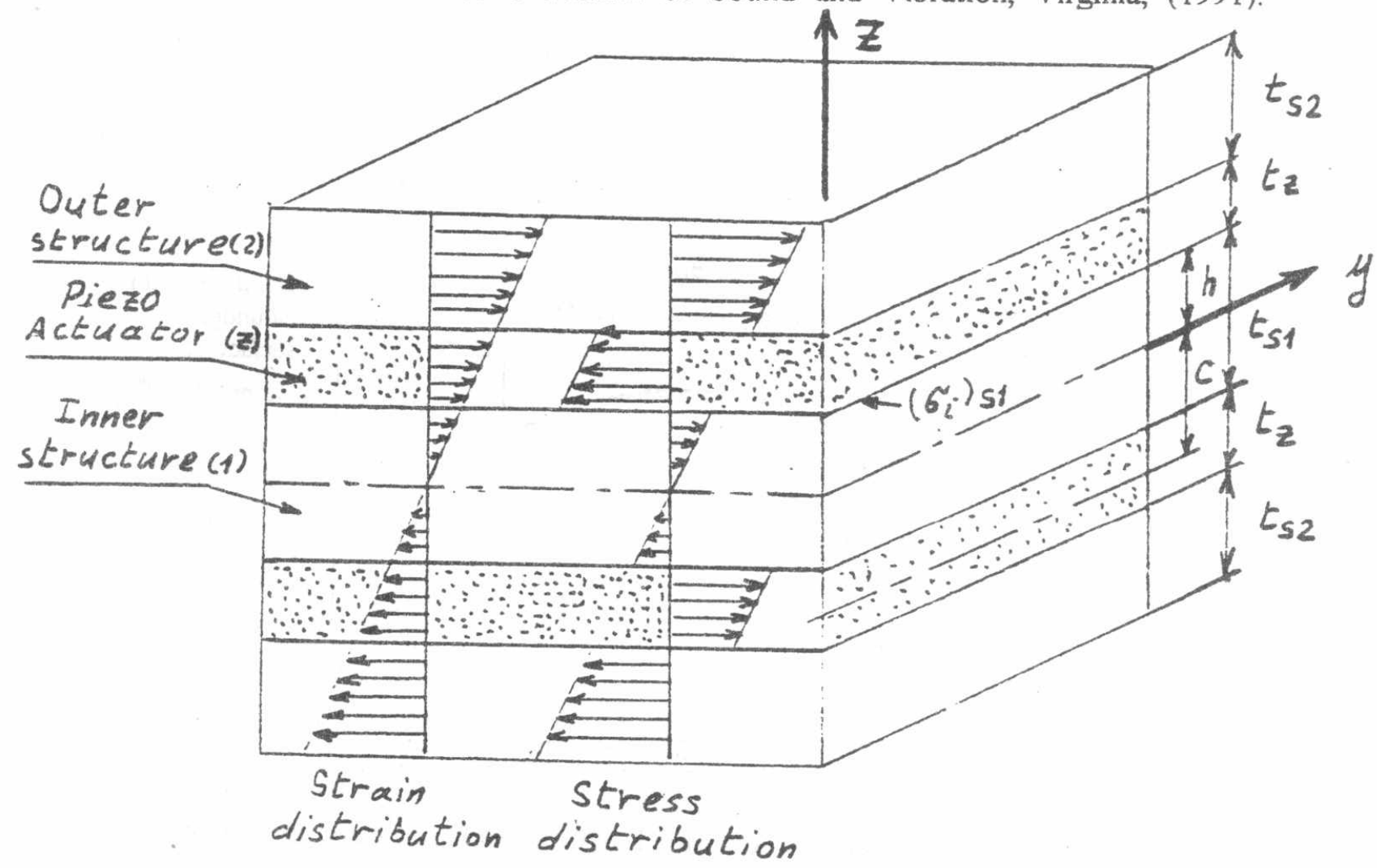

Figure 1: Dimensions of Layered Composite Structure and assumed Stress and Strain Distributions. 


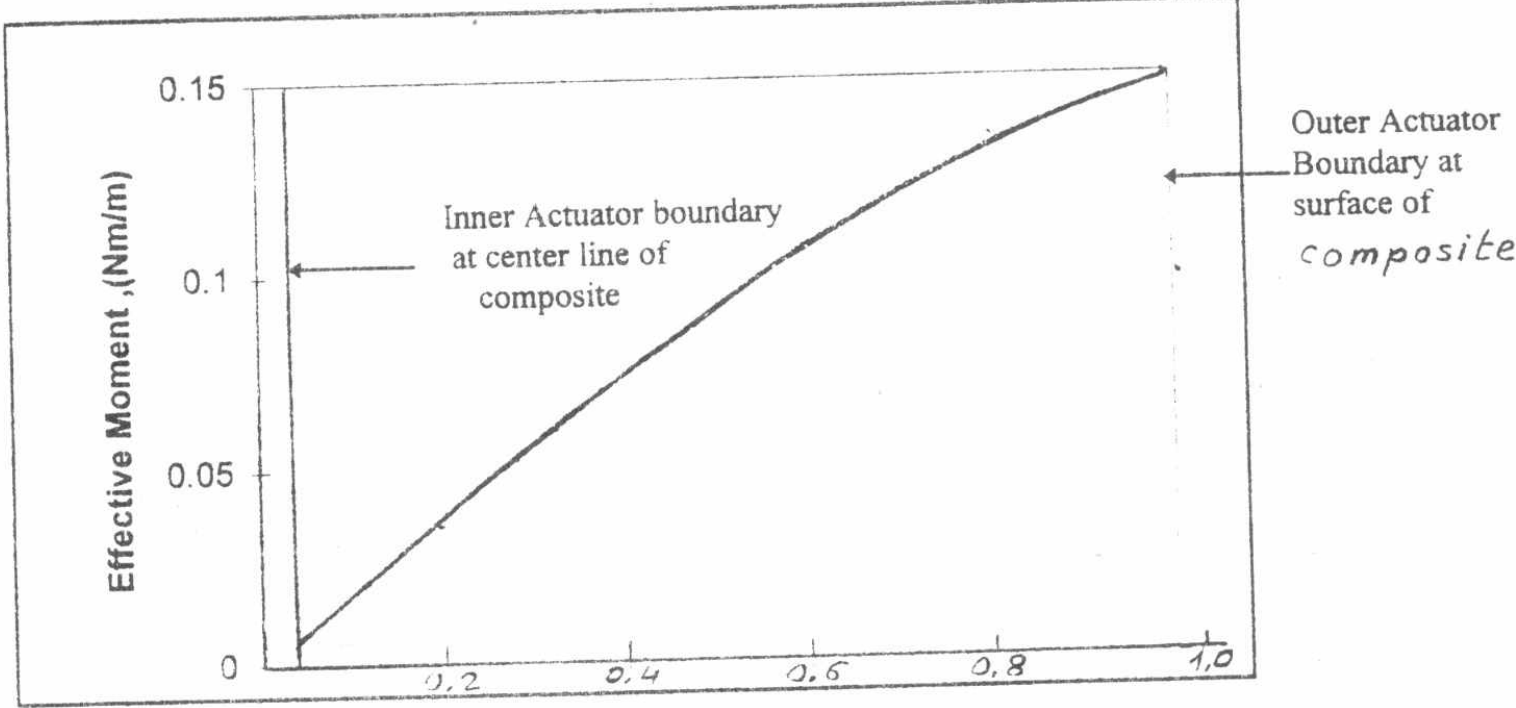

Non-Dimensional Actuator Position, $\rho_{\mathrm{c}}$

Figure 2: Effective Moment vs. Actuator Position for Thin Actuators, $\rho_{z}=0.05$

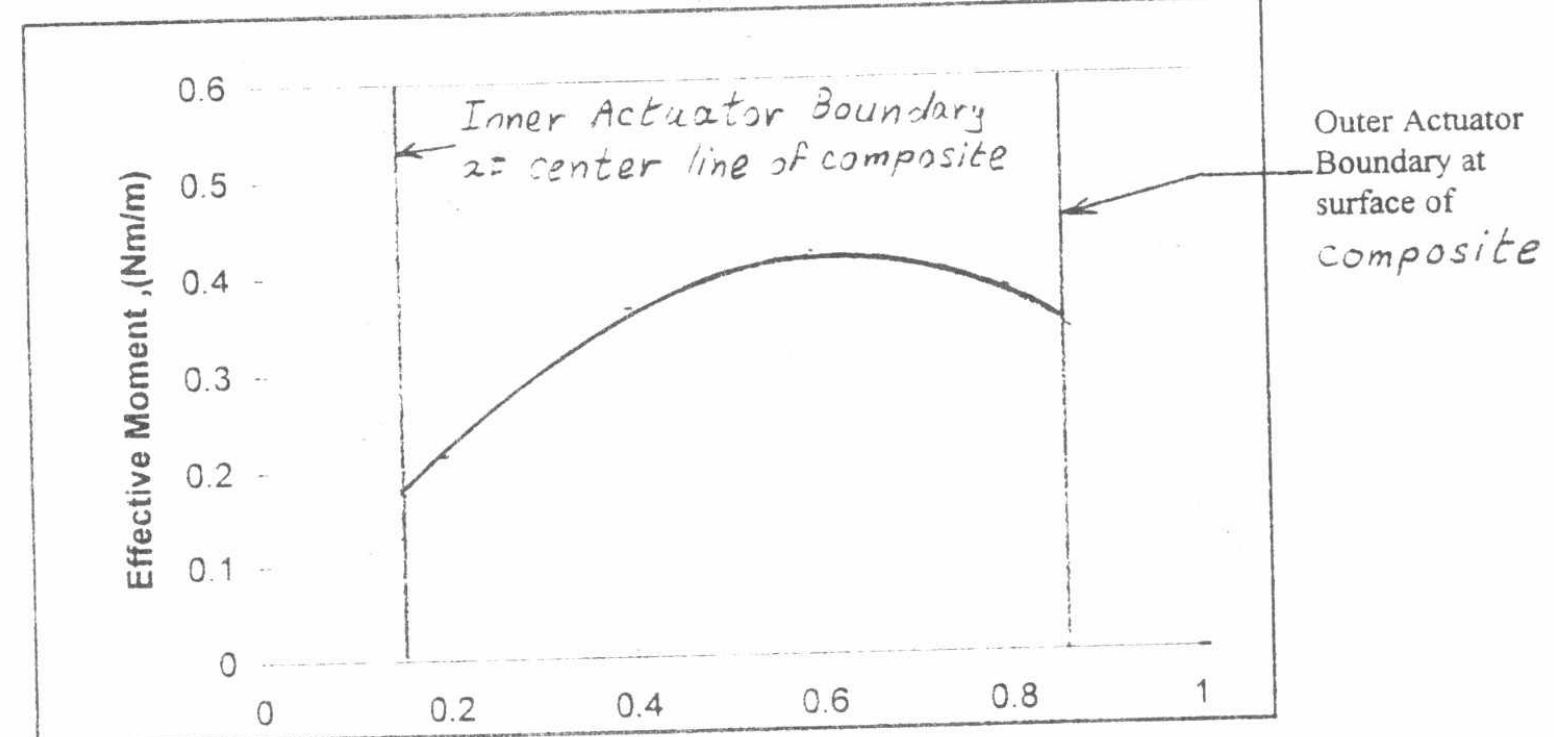

Non-Dimensional Actuator Position, $\rho_{c}$

Figure 3: Effective Moment vs. Actuator Position for Thick Actuators, $\rho_{z}=0.3$ 


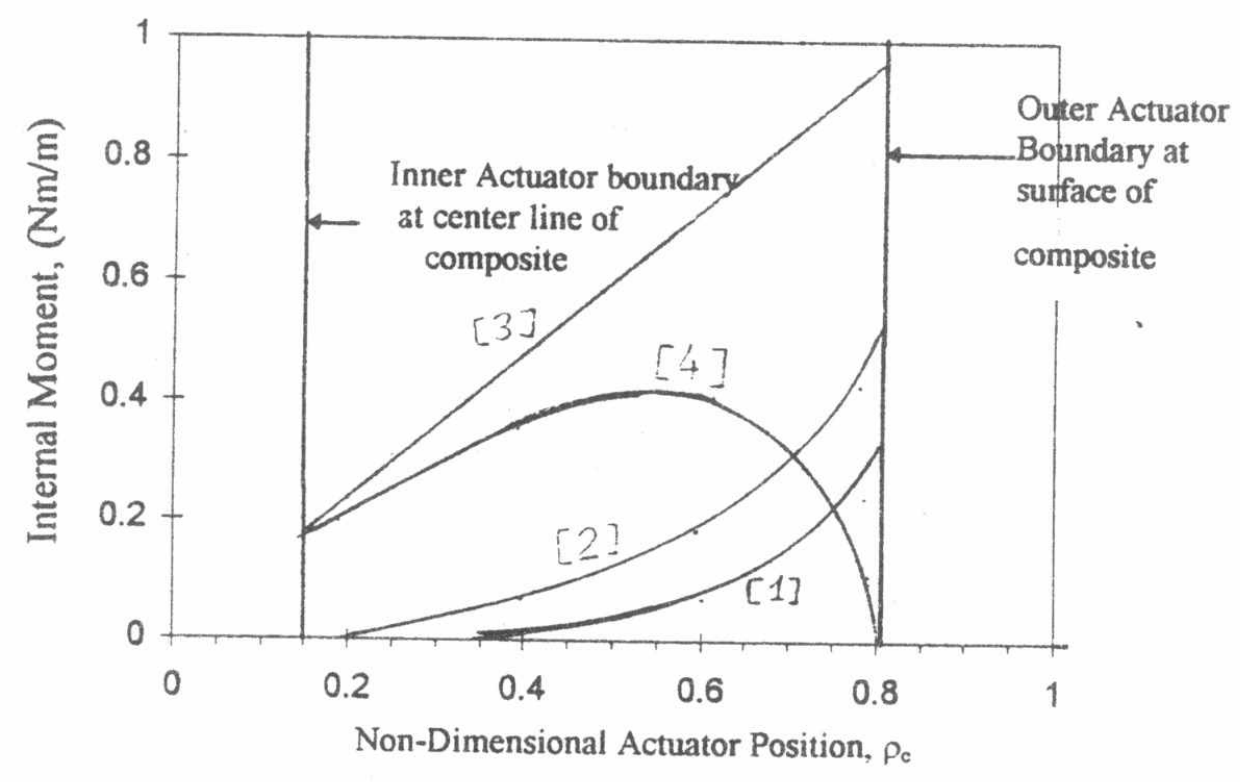

Figure 4: Internal Moments vs. Actuator Position for an Actuator Thickness of $\rho_{z}=0.3$

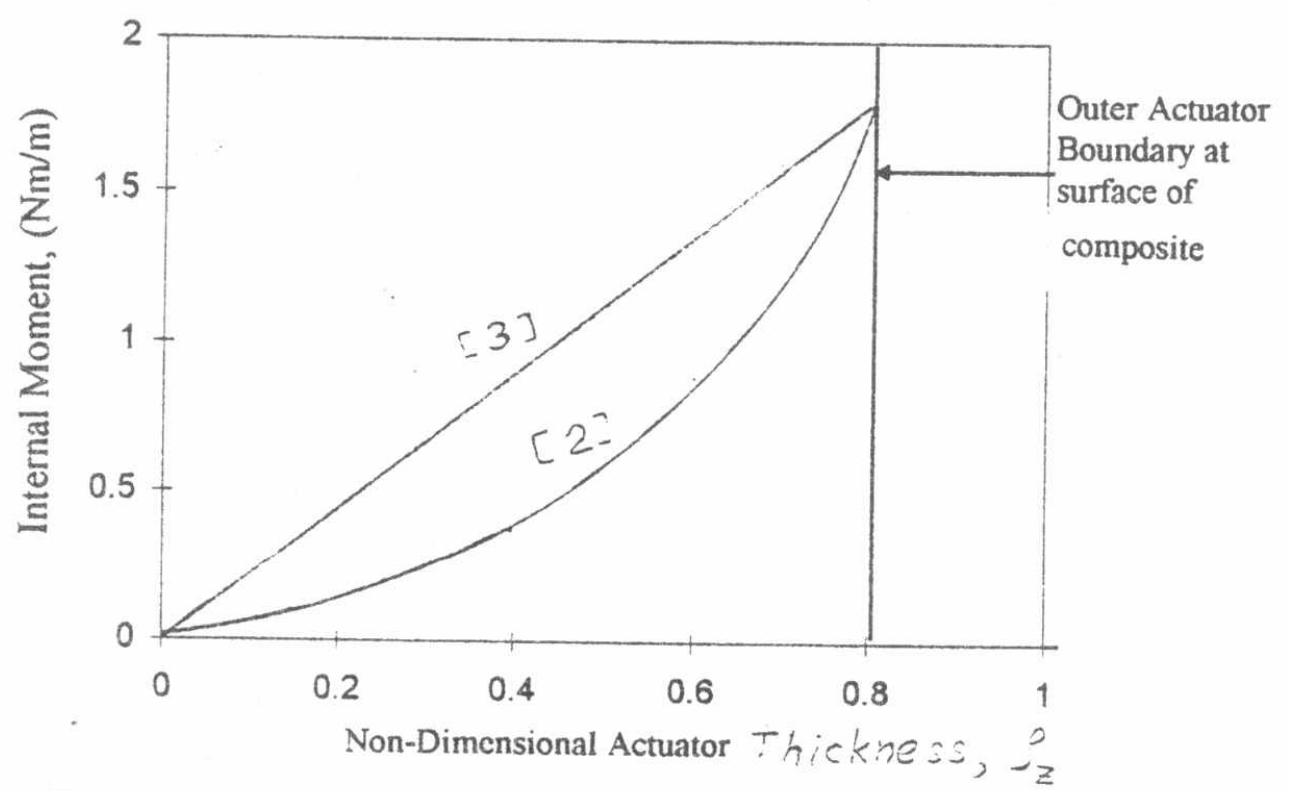

Figure 5: Internal Moments vs.Actuator Thickness for an Actuator Position of $\rho_{c}=0.6$ 